Tarbawi Ngabar: Jurnal of Education, Vol. 2. No. 2, Juli 2021, pp. 209-231

DOI 10.51772/tarbawi.v2i2.132, ISSN 2716-196X

Received: Mei 2021; Revised: Mei 2021

Accepted: June 2021; Published: July 2021

\title{
Pembelajaran Bahasa Arab di Kelas Bilingual dan Kelas Bina Prestasi di MTsN Ponorogo
}

\author{
Ratna Utami Nur Ajizah, M.Pd
}

Institut Agama Islam Riyadlotul Mujahidin Ngabar Ponorogo

ratnautaminurajizah@gmail.com

ملاخص

تعليم اللغة العربية وفقا بتحسين نوعية مؤسسات التعليمية الإسلامية التى تعد كعماد من العمود لترقية

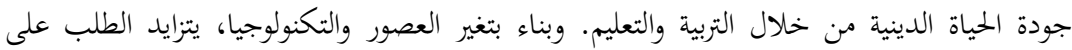

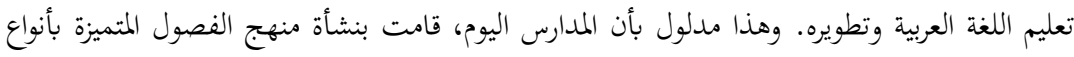

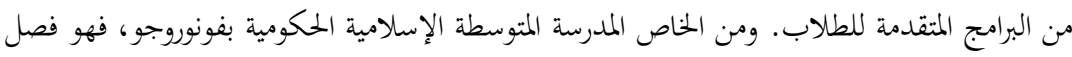
الثنائية اللغوية في المدرسة المتوسطة الإسلامية الحكومية 2 فونوروجو وفصل تدريب المدرسي التفوق في المدرسة المتوسطة الإسلامية الحكومية 3 فونوروجو. الكلمات المفتاحية: تعليم اللغة العربية، فصل الثنائية اللغوية، وفصل تدريب التفوق

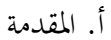

لا يختلف اثنان في أن اللغة العربية تحتل مكانًا شريفًا في المجتمع الإندونيسي وأن تعليمها وتعلمها

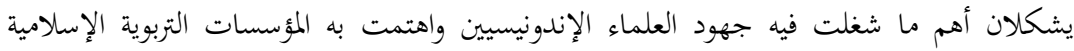
الإندونيسية. ولعل أهم ما يقف وراء ذلك كون إندونيسيا أكبر دولة إسلامية سكانًا فمن الطبيعي أن النداء يهتم الشعب الإندونيسي الذين كانت أغلبيتهم مسلمين اهتماهًا كبيرًا بهذه اللغة التي تنطق بها المصادر الأساسية لدينهم الإسلام.

1 مُمَّان حفص، منهج تعليم اللغة العربية لطالب البكالوريوس في قسم تعليم اللغة العربية، (رسالة الماجستير: جامعة مولنا

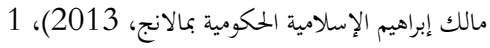


إلا أن هذا المكان العظيم للغة العربية والاهتمام الكبير بتعليمها وتعلمها لا يعني بالضرورة أن هذه اللغة قد حظيت ما تستحقه كلغة دينية وأجنبية من هندسة وسياسة التعليم المتقدمة. فإن الوضع الآتي لمجال تعليم اللغة العربية في إندونيسيا عبارة عن مرحلة يمكن وصفها انتقالية حيث أنه بدأ يشهد بعض التطورات الملحوظة الواعدة من ناحية، ولا يزال يتعرض لعدة مشاكل شائكة من ناحية أخرى. ومن بين تلك المشاكل هو عدم تحقق أهداف التعليم (أي تعليم اللغة العربية بوصفها اللغة الثانية) بالنسبة إلى مستوياتما اللغوية التي تختلف باختلاف المؤسسة الحاضنة لبرنامج اللغة، وهذه أشهر تقسيمات المستويات وفق أشهر وأهم 3 أطر عالمية، هي: معايير المجلس الأمريكي للغات، الإطار المرجعي الأوروبي المشترك، معايير الطاولة المستديرة، المستويات بحسب البرامج والجامعات الأخرى. المنهج الدراسى يتعلق بكل المراحل المدرسية وهو المرحلة الإبتدائية والمتوسطة والثانوية، في إندونسيا تنقسم فيها المدرسة الحكومية أو الإسلامية إلى ثلاث مراحل: وهي المرحلة الإبتدائية (SD/MI) والمتوسطة (SMP/MTS) والثانوية (SMA). ومدة الدراسة في المرحلة الإبتدائية ست سنوات، وفى المرحلة المتوسطة والثانوية ثلاث سنوات وهي الفصل الأول (الصف السابع فن المرحلة المتوسطة والعاشر فن المرحلة الثانوية) والثاني (الثامن فن المرحلة المتوسطة والحادي عشر في المرحلة الثانوية) والثالث (التاسع في المرحلة المتوسطة واثنا عشر في المرحلة الثانوية).2 والنّاظر إلى واقع تعليم اللغة العربية في المرحلة المتوسطة يجد أن معظم المدارس فشلت في تحقيق الأهداف السابقة، من أجل ذلك بدأت بعض المدارس في إبجاد الفصول المكثفة لترقية مهارة اللغة لدي طلاجها، من تلك المدارس هي المدرسة المتوسطة الإسلامية الحكومية 2 فونوروجو التي لها فصل الثنائية اللغوية (Billingual Class)، والمدرسة المتوسطة الإسلامية الحكومية 3 فونوروجو التي لما فصل تدريب التفوق (Kelas Bina Prestasi). لارتقاء جودة التعليم العربية الجيّدة بطرق مختلفة، خاصة في تحسين جودة تقنية الطلاب على اللغة العربية، ومن احدى طرقها هي تنظيم فصل الثنائية اللغوية وفصل تدريب التفوق، وهو الفصل الذي يستخدم لغة التدريس باللغتين العربية والإنجليزية، والذي يستخدمها في المدارس المتوسطة. وهذا فصل الثنائية اللغوية حل للمشكلة الرئيسية في تحسين القدرة التنافسية في العالمي. سيقوم هذا الفصل بتزويد

2 مملوءة النعمة، منهج تعليم اللغة العربية في المرحلة المتوسطة ، (أستاذة في جامعة زين الحسن الإسلامية غنغوغ

فروبولنجوا، 2013)، 5. 
الطلاب بمهارات الاتصال الشفوي والكتابي باللغتين الإنجليزية والعربية والتي ستكون مفتاحا لإتقان العلوم والتكنولوجيا لدى الطلاب. 3 ليس فقط من فصل الثنائية اللغوية، الذي يكون الحلول لمشكلات الرئيسية لتحسين القدرة التنافسية

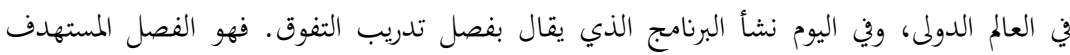
الخاص لطلبة الذين لديهم الإرادات والقدرات على تعزيزهم العميق لأجل تحقيق الأهداف المرجوة.

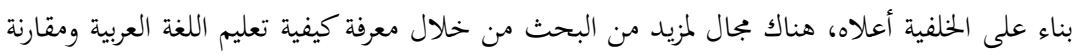

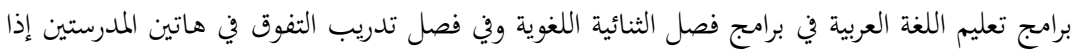

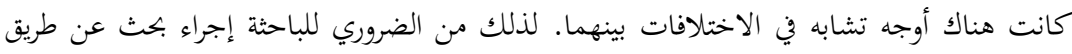

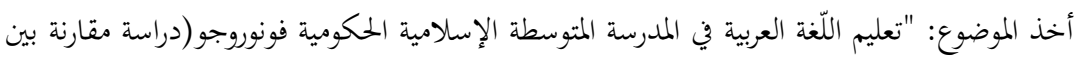

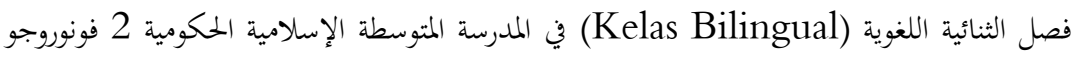
وفصل تدريب التفوق (Kelas Bina Prestasi) في المدرسة المتوسطة الإسلامية الحكومية 3

$$
\text { 1 ب. 1. تعلوروجو)". }
$$

تعليم اللغة العربية إنما هو عملية ذهنية واعية لاكتساب السيطرة على الأماط الصوتية والنحوية

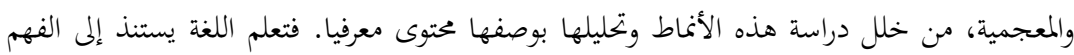

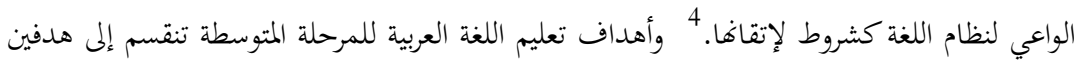

أهداف العامة: تعريف الطالبات بأهم طرق التدريس العامة ومعايير اختيارها. إلمام الطالبات بمفهوم اللغة بصفة عامه وخصائصها ووظائفها. إلمام الطالبات بأهم الطرق والاتحاهات الحديثة في تعليم اللغة. تعريف الطالبات بأهداف تدريس اللغة العربية في المرحلة المتوسطة ومناهجها. تعريف الطالبات بمهارات

${ }^{3}$ Dokumentasi Kelas Bilingual MTs Negeri 3 Ponorogo ( الوثيقة لفصل تدريب التفوق

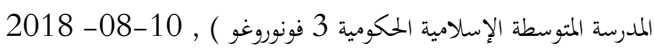

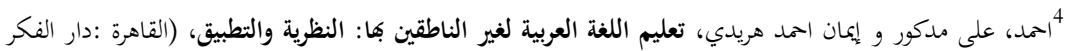

العربي، 2007)، 18. 


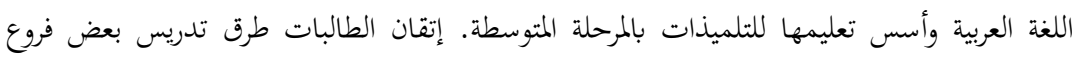

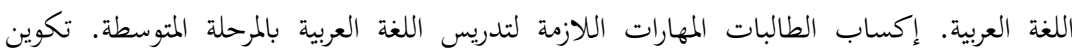

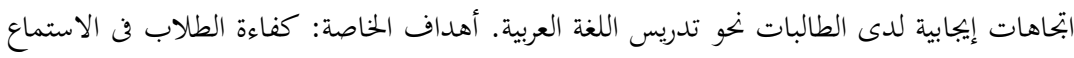

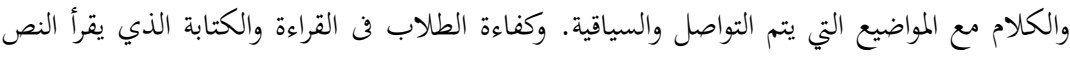

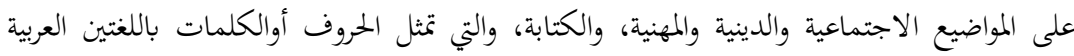

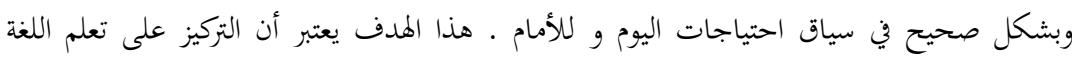

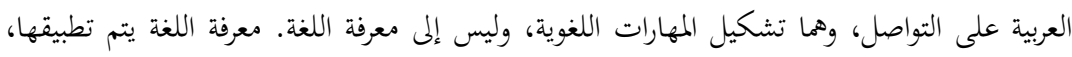
وليس النظري.

2. المناهج في تعليم اللغة العربية للمرحلة المتوسطة

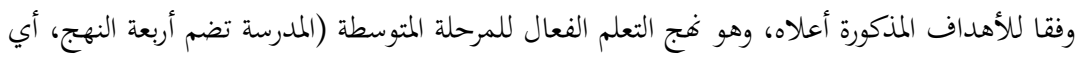
هج إنساني، التواصلية، السياقية، والهيكلية). أ) منهج إنساني أي نرى أن تعلم اللغة العربية يتطلب الطلابية النشطة، وليس أعضاء هيئة التدريس.

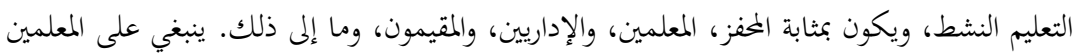

$$
\text { الاستفادة من جميع الطلاب المحتملين. }
$$

ب) منهج التواصلي أن نرى أن الوظيفة الأساسية للغة هي التواصل. وهذا يعني أن اللغة العربية ينبغي

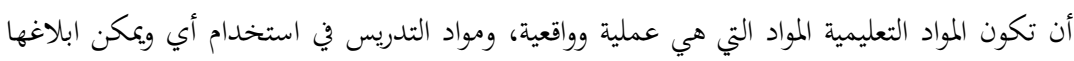

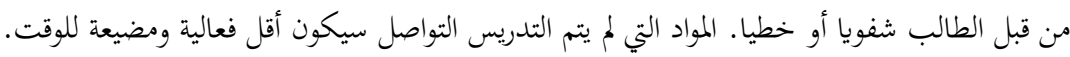

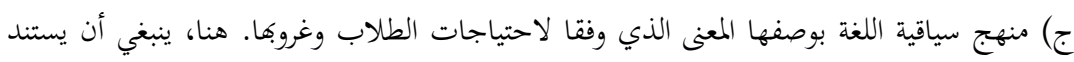
تصميم المواد التعليمية على احتياجات وكالة، واحتياجات الطلاب اليوم وإلى المستقبل.

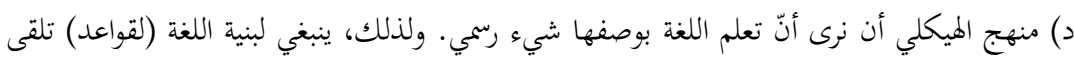

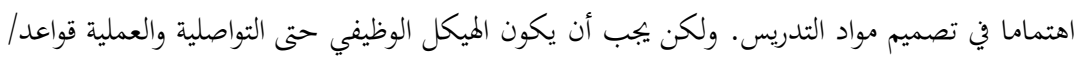

5

http://www.kau.edu.sa/content.aspx?Site_ID=0009314\&lng=AR\&cid=27403 
النحوي ليس من العملي وليس التواصلية في تعلم اللغة العربية قد فشلت في تشكيل لطلاب اللغة المهرة، وليس فقط العربية بل أيضا الانكليزية. 6 3. طرق تعليم اللغة العربية

الطريقة هي النظام الذي يسير عليه المدرس في إلقاء درسه ليوصل المعلومات إلى أذهان التلاميذ بشكل يحسن أغراض التربية. وطريقة التعليم ينبغي أن ينظر إليها لا على أهما شيء منفصل عن المادة العلمية

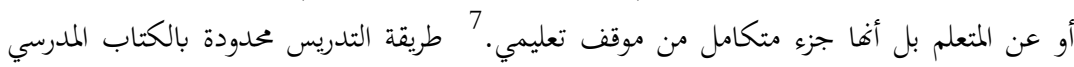
ويكون معيارا للتدريس. والمدرس يتأثر بطريقة التعليم التي يمكن أن تتمشى مع الكتاب المدرسي، والطالب يتأثر بطريقة التعليم التي تستفاد من طريقة الكتاب المدرسي. 8 وأما الطرق المستخدمة لتعليم اللغة الأجنبية فهي: طريقة القواعد والترجمة، فالمدرس ينطلق من القواعد النحوية التي يكلف التّلاميذ بحفظها. وبذلك فإن الدرس عبارة عن نص أو مجموعة من النصوص التي يتدرب التّلاميذ على ترجمتها بادئا بالمفردات، ثم الجمل، ثم النصوصكاملة،ويلاحظأن التركيز هنا على القراءة والكتابة والترجمة. ثم الطريقة النفسية أو السيكولوجية (Psychological Method) وهي طريقة تقوم على تعليم المفرداتأولا من خلال بناء سلسلة من الجمل تدور حول أنشطة الحياة اليومية والمواقف العملية منها مثل الاستيقاظ في الصباح وتناول الطعام والذهاب إللالسوقوزيارة الطبيب والآخر. 10 وتليها الطريقة الصوتية (Phonetic Method) وهي الطريقة في المرحلة الصوتية للطريقة المباشرة، وهي كالطريقة السيكلوجية تركز على الممارسة الشفهية أولا، إلا أها تستخدم طريقة علمية منطقية كمدخل للمشكلة، فهي تبدأ بدراسة الجهاز الصوتي وطريقة إخراج الأصوات، وقبل بدء دراسة اللغة

${ }^{6}$ http://afrizaldaonk.blogspot.com/2011/01/blog-post_3796.html 7 حسن شحاتة، تعليم اللغة العربية بن النظرية والتطبيق، (مصر: المكتبة المصرية اللبنانية، 2008)، 20 8 رشدي أحمد طعيمة ومحمود كامل الناقة، تعليم العربية لغير الناطقين بها، الكتاب الأساس الجزء الأول، (مكة المكرمة: جامعة أم القرى، 1983)، 9علي أحمد مدكور وإيمان أحمد هريدي، تعليم اللغة العربية لغير الناطقين بها النظرية والتطبيق،(القاهرة: دار الفكر العرب، . 2007)، 2007 10 10لمرجع نفسه، 
يتعلم التلاميذ الطريقة السليمة لتشكيل وإخراج كل الأصوات، كما يتعلم ونقراءة أصوات الحروف الأبجدية وكتابتها.

والطريقة الطبيعية التي تعتمد على عدم استخدام اللغة الأم في قاعة الدرس تقوم على أساس أن الفرد

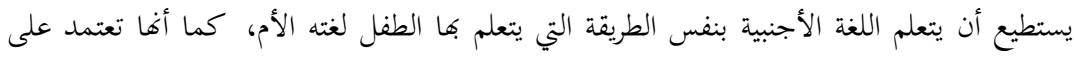
التمثيل الصامت والترديد والأسئلة والإجابات المتبادلة كوسيلة لفهم اللغة وتقليدها. والمفردات في هذه لتهني الطريقة تكتسب من خلال الأنشطة اليومية. 11 ثم طريقة القراءة (The Reading Method) التي ترى أن التخيل السمعي للغة التي يمصلها

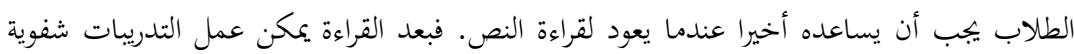

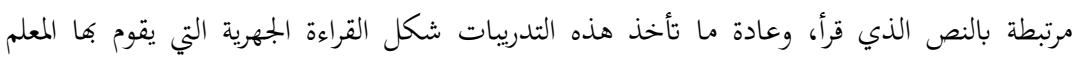

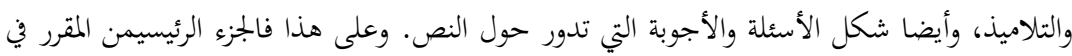
هذه الطريقة يخصص للقراءة المركزة والواسعة. 12 الطريقة السمعية الشفوية (The Audio Lingual Method) هي التي توجب في تعليم اللغة

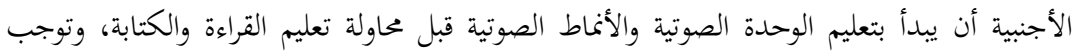

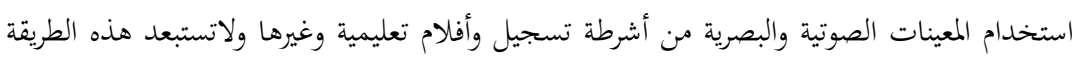
الاستعانة باللغة الأصلية. 13 أما الطريقة التوليفية أو الانتقائيةفقد عرفتها (Eclectic Method) بأها طريقة المعلم الخاصة التي بأية يستفيد فيها من كل عناصر الطرق الأخرى التي يشعر أها فعالة. وهذه الطريقة عادة ما تتغير مع كل

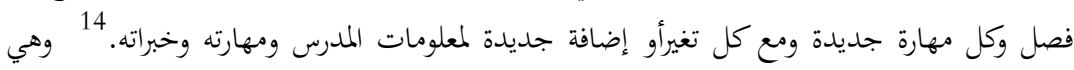

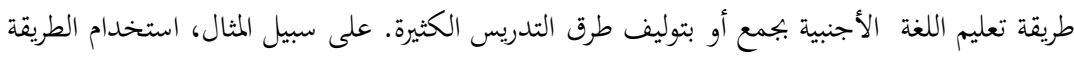

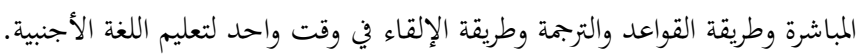

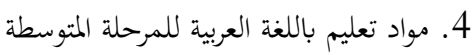

11 مممود كامل الناقة، تعليم اللغة العربية للناطقين بلغات أخرى، (مكة المكرمة: جامعة أم القرى، 1985)،.77 77 12 13 علي الحديدي، مشكلة تعليم اللغة العربية لغير العرب، (القاهرة: دار الفكر العربي، 2005)، 5.

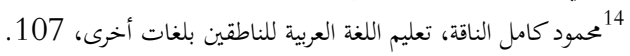


دراسة المواد العربية تتكون من مكونات اللغة والمهارات اللغوية وعناصر اللغة، وهي تتكون من المفردات والأصوات والهياكل. وتتكون المهارات اللغوية من الاستماع والكلام والقراءة والكتابة. إن المستوى 15

المتوسط يعبر المرحلة تثبيت هذه المهارات الأساسية وتوسيع نطاقها وزيادة الثروة اللغوية عند الطالب. ووفقا للدكتور عبد الحليم حنفي "موضوعات اللغة العبية هي مواد التدريس الفعالة هي المواضيع التي التواصلية والسياقية حول موضوعات الحياة اليومية، والدين والعلوم والتكنولوجيا". يقدم هذا المقرر المفاهيم والمهارات الخاصة بتدريس اللغة العربية في المرحلة المتوسطة، فيمهد لذلك بطريقة التدريس من حيث مفهومها وأنواعها، ومعايير اختيارها، ثم يعطي فكرة موجزة عن تعليم اللغة بصفة عامة، ثم يتناول اللغة العربية من حيث أهمية تعليمها ونشرها، وخصائصها، ومهاراما في التعليم العام، كما يقدم طرق تدريس كل من الإستماع والكالام والقراءة والكتابة، على أن يتم استكمال بقية فروع اللغة العربية في المقرر الآخر.

5. تقويم تعلم اللغة العربية للمرحلة المتوسطة أ) أختبار كتابي

الشركة المصرية للاتصالات هو الاختبار التحريري الذي سيلة للرد على أسئلة أو لا مشاكل في الكتابة • وهذا هو، على الإجابة التي قدمها على شكل مكتوب بلغة بصدد إجراء تجارب. في غضون ذلك، كيفية كتابة الأسئلة يمكن تشكيلها ويمكن أيضا أن يكون عن طريق الفم، مثل الاستماع والاختبارات الاملاء. وهذا يعني، أنه حتى لو كان ذلك يعني إعطاء الأسئلة الشفوية، ولكن الجواب الذي قدمته لـنه بصدد إجراء بحارب على شكل مكتوب، ثم اختبار يسمى اختبار كتابي. ب) اختبار شفوي

الاختبار الشفوي هو اختبار لكيفية الإجابة عن الأسئلة أو المشكلات القيام به شفويا. هذا الاختبار الشفوي هو مناسبة لقياس القدرة على الكلام (الكلام). من خلال هذا الاختبار عن طريق الفم ، يمكن أن تعرف قدرة الطلاب على القدرة على الحديث، سواء من الجانب من لهجته، النحوية، وساسة، والمراسيم اختيار الكلمات، اسلوبها، والدقة في تقديم المعلومات، والضغط، وبلاغة في لفظ كلمة. أيضا قياس القدرة على الكلام، ويمكن أن يتم تنفيذ الاختبارات الشفوية لقياس القدرة على

15 رشدي أحمد طعيمة، تعليم العربية لغير الناطقين بها، (الرياط: منشورات المنظمة للتربية والعلةم والثقافية "إيسيكا"،

. 1417هـ/ 1499 1999م)، 47 
قراءة النصوص العربية. من خلال هذا الاختبار الشفوي، والقدرة، ليس فقط بصدد إجراء تجارب معروفة من اختصاص فهم النص، ولكن المعروف أيضا من الجانب من اختصاص بصدد إجراء بتارب على إتقان القراءة والطلاقة في نطق الكلمات ، والتجويد والدقة وسرعة القراءة. 16 ج. مفهوم فصل الثنائية اللغوية إن مصطلح الثنائية اللغوية يرادفه في اللغة الإنجليزية مصطلح Bilingualism. ولقد خطر ببالي أن أترجم هذا المصطلح الأجنبي بعدة وجوه منها: 1. ثنائية اللغة: ولقد عدلت عن هذا المصطلح أيضا للسبب ذاته، حيث أنه قد يدل على أن اللغة ذاتما ثنائية. الثنائية اللغوية هي استعمال الفرد أو الجماعة للغتين بأية درجة من درجات الإتقان ولأية مهارة من مهارات اللغة ولأي هدف من الأهداف. 17 لقد استخدم مصطلح التعليم الثنائية اللغوية ليؤدي معاني مختلفة. فلقد استخدمه البعض ليدل على أية مدرسة فيها لغتان ومنهما لغة أجنبية. وهذا يعني أن كل مدرسة تعلم لغة غير اللغة الأولى توصف بأن فيها تعليما الثنائية اللغوية. وبذلك تصبح معظم برامج التعليم في العالم من هذا النوع، لأن معظم البرامج، إن لم تكن جميعها، تعليم لغة أجنبية واحدة أو أكثر في مدارسها. والمثال جميع المدارس المتوسطة والثانوية في البلاد العربية تعليم اللغة الإنجليزية أو اللغة الفرنسية، يدل هذا المثال على تعليم الثنائية اللغويةكما في هذا التعريف. 18

د. مفهوم فصل تدريب التفوق (Kelas Bina Prestasi)

لن يتوفق الجهود المبذولة لتحسين وتنمية نوعية التعليم. وشارك أنواع البرامج المبتكرة في تنشيط بتديد التربية، منها الفصل المتميز. وكان فصل تدريب التفوق كنوع من أنواع الفصل المتميز يستملك الخصائص المماثل به. فيحتاج التعليم المختلف لتلبية احتياجات الطلبة الموهوبين من حيث توفير المواد التعليمية لتناسب اهتمامات الطلبة ومواهبهم وقدراتم الفكرية. مما يمكن لهؤلاء الطلبة على أن يشعروا

16 M.Ainin, dkk, Evaluasi Pembelajaran Bahasa Arab (تقويم تعلم اللغة العربية) , (Malang: Misykat, 2006), 115-116

$$
\begin{aligned}
& \text { 17 18حّّم على الخولي، الحياة مع لغتين ( الثنائية اللغوية)، ( مكة :جمع الحقوق محفوظة للمؤلف، 1988)، } 18 . \\
& \text { 18 }
\end{aligned}
$$


سهولة من أنه لم يتم شحذهم بالمنهج الدراسية العادية. 19 فيحتاج الطلبة الموهوبون إلى اهتمام و عناية خاصة للتغلب المشكلات الموجهه في تحقيق مواهبهم المتفوقة. كان فصل تدريب التفوق فصلا متميزا، حيث الطلاب فيه من الطلاب الذين لديهم الانجازات المتميزة ثم جمعهم في فصل خاص. 20 هـ. واقع تعليم اللّغة العربية في فصل الثنائية اللغوية في المتوسطة الحكومية 2 فونوروجو وفي فصل تدريب التفوق في المدرسة المتوسطة الحكومية 3 فونوروجو 1. تعليم اللّغة العربية في فصل الثنائية اللغوية في المتوسطة الحكومية 2 فونوروجو أ) أهداف تعليم اللغة العربية ويحتوي أهداف تعليم اللغة العربية فصل الثنائية اللغوية بالمدرسة المتوسطة الحكومية 2 فونوروجو: على تنمية كفاءة لغة الطلاب في مهارة اللغة الأربعة، الإمام بعناصر اللغة العربية، والمعرفة على ثقافة العربية، والتعويد على الكالام باستخدام اللغة العربية. بنظر إلى أهداف في فصل الثنائية اللغوية بالمدرسة المتوسطة الحكومية 2 فونوروجو مطابقا في بحث السابق المذكر في الأول، على الرغم من عدم وجود كله، إلا أن مستوى النجاح في تحقيق الأهداف أثبت نجاحه من نتائج البحث في مهارة الكلام جيد. يقدر الطلاب على فهم المسموع من كلام الأستاذ في التعليم ويقدر الطلاب على الكتابة من السمع مدرسه بالصحيح. ب) المناهج تعليم اللغة العربية نفذ تعليم اللغة العربية في فصل الثنائية اللغوية باستخدام المنهج الدّراسي 2013. إذ أكّد هذا المنهج الدراسي على التوازن بين الموقف أو السلوك والمعرفة والكفاءة. وأما المنهج الدّراسي في فصل الثنائية اللغوية مناسبة بالمنهج المدرسة المرحلة المتوسطة. عملية تعليم مهارة اللغة العربية في المدرسة المتوسطة الإسلامية الحكومية 2 فونوروجو خاصة لفصل الثنائية اللغوية إلى نوعين، هما كما يلي: 1) عملية التعليم داخل الفصل الدراسي (الأنشطة الأكادمية)، أي تنفيذ عملية تعليم مهارة اللغة العربية داخل الفصل الدراسي مند ساعة 06.45 إلى 14.35. ولكن تنقسم الفرصة الدراسية إلى

${ }^{19}$ Daniel Muijs, David Reynolds, Effective Teaching Teori dan Aplikasi, ter. Helly prajitno soetjipto dan Sri Mulyantini Soetjipto (Yogyakarta: Pustaka Belajar, 2008), 261.

$$
\text { 20 } 262
$$


عدة المواد الدراسي المختلفة، وبنسبة إلى الفرصة الخاصة لتعيلم مهارة اللغة العربية ضيقة. إذ في النهاية يكون مفموم الطلاب نحو الحوار باستخدام اللغة العربية ضعيفة، ولم يقدر الطلاب أن يقوم بالمحادثة اليومية باستخدام اللغة العربية الجيّدة.

2) الأنشطة اللغوية خارج الفصل الدراسي (الأنشطة غير الأكادمية)، أي الأنشطة اللغوية النافذة خارج الفصل الدّراسي إضافية للطلاب في المدرسة المتوسطة الإسلامية الحكومية 2 فونوروجو خاصة لفصل الثنائية اللغوية. وأصبحت هذه الأنشطة استمرارية من تنفيذ فصل الثنائية اللغوية، لأنّ الأهداف من إيجاد هذا الفصل هو إلمام الطلاب نحو المهارة اللغوية الأربعة، خاصة فن مهارة الكلام حتى يستوعب الطلاب على استخدام اللغة العربية في المحادثة اليومية. تتكون الأنشطة اللغوية من ثمانية البرامج، وتعدّ فن أوقات مخصوصة لتطوير كفاءة لغة الطلاب. 21 ج) طرق تعليم اللغة العربية استخدام طرق التعليم أو استراتيجية التعليم غير المناسب بماكتب فن إعداد التدريس ويؤدي هذا الواقع إلى عدم الحماسة من جانب الطلاب. وأما طرق التعليم المستخدمة بالمدرسة المتوسطة الحكومية فونوروغو تركز في تطبيق طرق التعليم الجارية في المنهج الدراسي 2013. إذ تشمل الطرق كما هي فن المنهج الدراسي 2013. ومن البحث، استخدام طريقة التعليم اللغة العربية في فصل الثنائية ...مطابقا بالنظرية، استخدام طريقة الإنتقائية مناسبة عند التعليم لأن لكل مادة اللغة العربية تقسم من المهارات ولا يستطيع المعلم أن يستخدم بالطريقة مساوية في تعليم اللغة العربية. وهذه الطريقة فعالة لسهولة فهم الطلاب وطريقة

$$
\text { دالإنقائية لا يجعل الطلاب بالشائم. }
$$

مادة تعليم اللغة العربية في مستوى المتوسطة بحمع في مادة واحدة يعنى اللغة العربية والكتاب المستخدم في فصل الثنائية اللغوية بالمدرسة المتوسطة الحكومية 2 فونوروجو مطابقا بالنظام الحكومية الموجودة. الكتاب المستخدم فصل الثنائية اللغوية بالمدرسة المتوسطة الحكومية 2 فونوروجو هو كتاب اللغة

21 سيتي، المقابلة 15، المدرسة المتوسطة الإسلامية الحكومية 2 فونوروغو، 30 مارس 2019. 
العربية على أساس المنهج الدراسي2013 من قبل وزارة الشؤون الدينية إندونيسيا. واستخدام مصادر 22 التعليم الممل يؤدي إلى قلة معارف الطلاب. هـ تقييم تعليم اللغة العربية

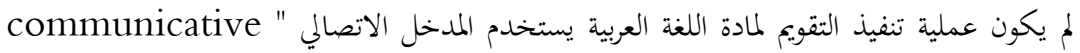
approach بصورة العامة. وبجانب ذلك، يظهر الإبهام بين المواد الدراسي و المؤشرات للتقويم، ومن ثم يظهر

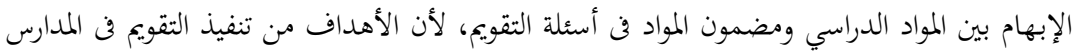

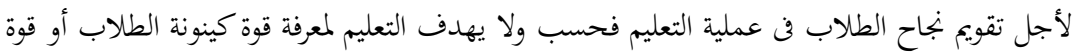

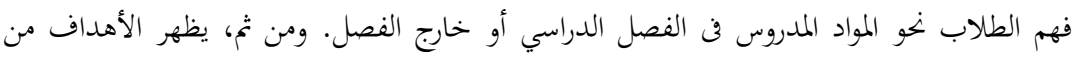

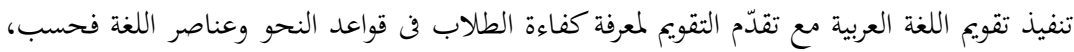

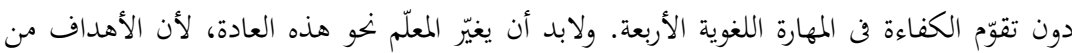

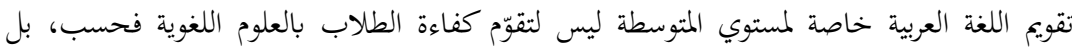

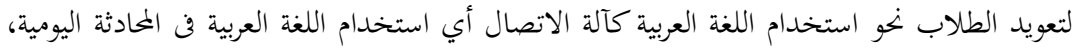
ويليه لتعويد الطلاب نهو المهارة الاتصالية بصورة الكلام والكتابة كما هى في مهارة اللغة الأربعة.

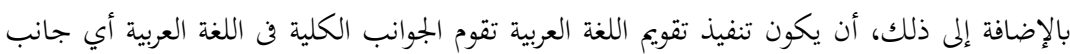
المعري والعاطفى والحركي.23

وبنسبة إلى ما علمت الباحثة من البيانات السابقة، فآرت الباحثة أن المعلم بالمدرسة المتوسطة

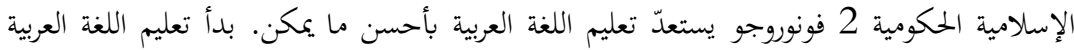

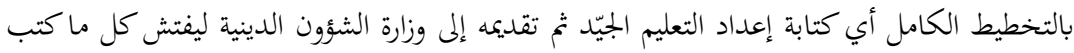
المعلم في إعداد التعليم. وبجانب ذلك، لابد على المعلم أن يكتب الأشياء المتعلقة بتنفيذ تعليم اللغة إلغ وراه

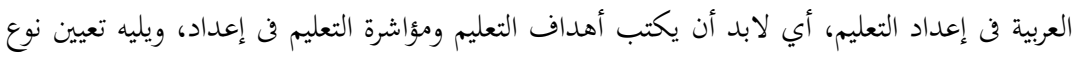
التقويم حسب أهداف التعليم المكتوبة.

2220يتي، المقابلة 17، المدرسة المتوسطة الإسلامية الحكومية 2 فونوروجو، 30 مارس 2019.

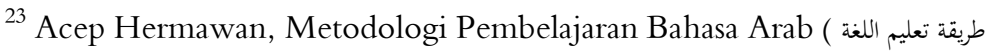
(العربية),(Bandung: Remaja Rosda Karya, 2011), 284 
إنما التقويمات المستخدمة في فصل الثنائية اللغوية هما التقييم الشفوي والتقييم التحريري. ومن المعلوم، أن التقويم هو الدور الآخير من عملية التعليم، ولكن يحتاج التقويم إلى التخطيط المطبوخ لعدة الأسباب التالية:

1) تخطيط تنفيذ التقويم حسب تطوير أهداف التعليم،

2) أصبح التخطيط لتنفيذ التقويم آلة لتفتيش أهداف التعليم حسب التخطيط الموجود فن إعداد التعليم6 3) أصبح التخطيط لتنفيذ التقويم دليلا على عملية التعليم حسب إعداد التعليم، 4) يدل التخطيط لتنفيذ التقويم أنواع الصعوبة والسهولة في عملية التعليم. ونظرا إلى أنواع البرامج بمدرسة المتوسطة الإسلامية الحكومية 2 فونوروجو الكثيفة، فيكون تنفيذ عملية التقويم موسوس ومنزعج. إذ يؤدي هذه الحالة إلى تأخر استعداد الطلاب عند عملية التعلم واستعداد التقويم. ويليه يؤدي هذه الأسباب إلى عدم نتائج الطلاب الممتازة ولم يصيل النتائج إلى حدّ

$$
\text { و الأقصي. }
$$

ومن البرنامج الداعمة للتعليم فصل الثنائية اللغوية في المدرسة المتوسطة الحكومية 3 فونوروجو مما يلى: صدور كتاب المفردات، حفظ المفردات مرة في الاسبوعين، سلامة قربان، مسابقة مهارة اللغوية، مغامرة اللغة، أسبوع العربي، تدريبات اللغة العربية لمدرسي العلوم الدينية، والجدار الإعلامي للمغردات.

2. تعليم اللّغة العربية في فصل تدريب التفوق في المدرسة المتوسطة الحكومية 3 فونوروجو إن منهج فصل تدريب التوقيف هو منهج خحص للطلاب المحتملين الذين للديهم المواهب والاهتمامات وإرادات التعليمي المثالي والعالي وإرشادات عالية للدراسة ومواصلة التعليم الأعلى. وإن لفصل تدريب التوقيف مميزات من الفصول العادية الأخرى، منها التمييز في مجال الامكانات وأوقات التعلم ومحتوى الدراسى ومدخل التعليم المستخدم بالمعلمين. تعليم اللغة العربية في فصل تدريب التفوق (Kelas Bina Prestasi) في المدرسة المتوسطة الحكومية 3 فونوروجو. أ) أهداف تعليم اللغة العربية 
إنما أهداف التعليم من فصل تدريب التفوق (Kelas Bina Prestasi) في المدرسة المتوسطة

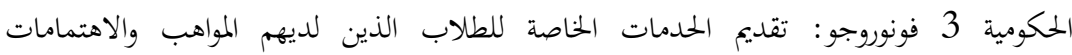
والامكانات العالية للتعلم مناسبة بقدرات الطلاب عن طريق تنمية قدرات التفكير شموليا وابتكاريا وإبداعيا وتنمية مهارات الطلاب في اللغة العربية، والقراءة، وتحفيظ القرآن الكريم، ممارسة الطلاب بأبداء صلاة الضحى والصلاة الجماعة، والتدريب وإعداد الطلاب لمشاركة الأولمبياد منطقيا أم وطنيا.

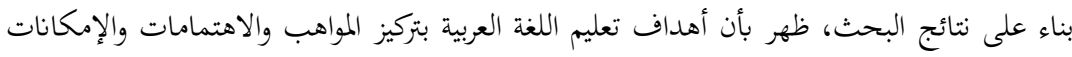

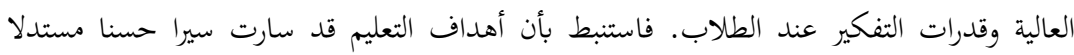
بالتواصل الأفضل للطلاب باللغة العربية، وتسهيل الطلاب في قراءة وحفظ القرآن الكريع.

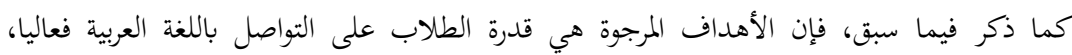

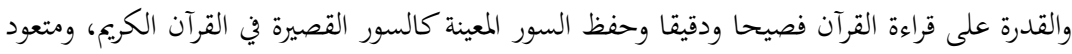

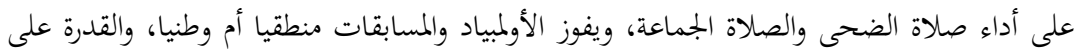
قراءة الكتب المعينة صحيحا ودقيقا. بناء على نتائج هذا البحث، ظهر بأن الأهداف المرجوة في تعليم اللغة العربية قد سارت سيرا حسنا.

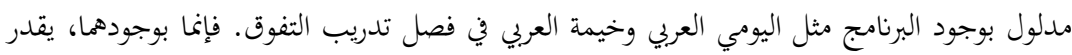

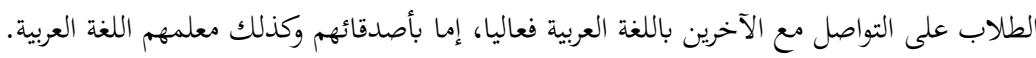

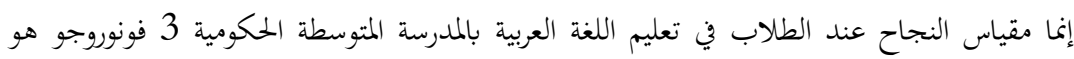

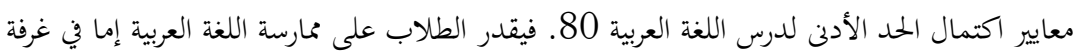

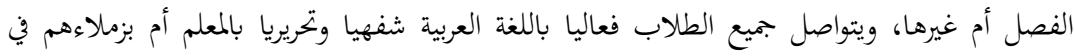
فصل تدريب النوقيف. بناء على نتائج هذا البحث بأن مقياس النجاح عند الطلاب في تعليم اللغة العربية يقال ناجحا. يمكن

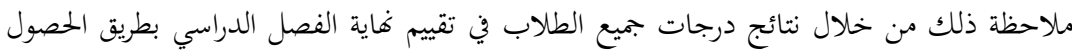

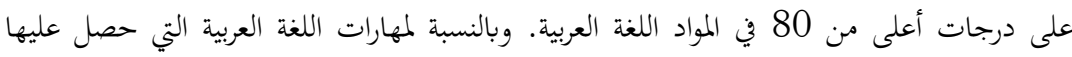
الطلاب في فصل تدريب التفوق، فسوف يستمرون في الزيادة إلى جانب مستوى الفصل.

$$
\text { ب) المناهج تعليم اللغة العربية }
$$

تعليم اللغة العربية في فصل تدريب ، فهو بتطبيق المنهج 2013. ويوجد الإضافية المعات في هيكال فصل

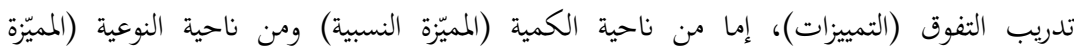


التنافسية). فإن تعليم اللغة العربية في فصل تدريب التفوق ثلاث حصص الدراسية وحصتين مسائيا في

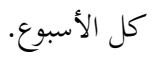
وبنسبة إلى البحث المنهج الدّراسي في فصل الثنائية اللغوية مناسبة بالمنهج المدرسة المرحلة المتوسطة. ولزيادة هيكال المنهج من المدير فصل تدريب التفوق ولا يزيد ولا ينقص الكفاءة الأساسية منهج الدراسي 2013 ، وهذا يؤدي إلى برنامج اللغوية والدينية والعلمية في فصل تدريب فئ لدريب التفوق. ج) طرق التعليم ومن طرق التعليم المستخدم في تعليم اللغة العربية في فصل تدريب التفوق بتركيز على مدخل التعلم

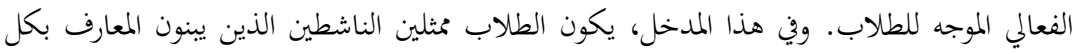

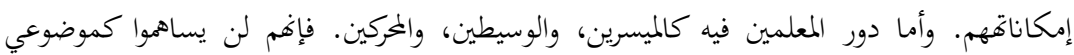

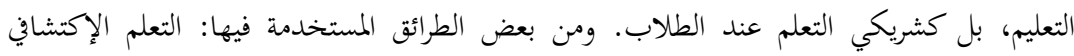

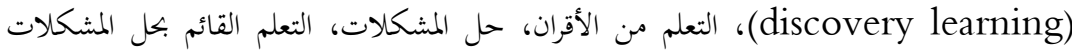
(problem based learning) بناء على نتائج البحث ظهر بأن طرق المدخل الموجه للطلاب يستنبط بطريقة التعليم الدقيقة. ويككن

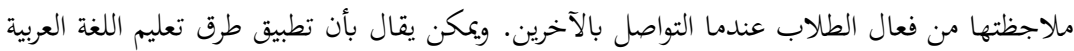
في فصل تدريب التفوق هو الطرق المختلطة/ الطريق الانتقائية.

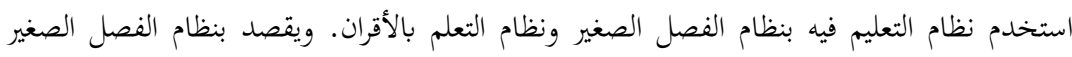

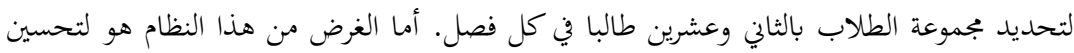

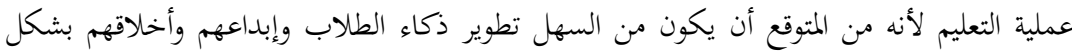

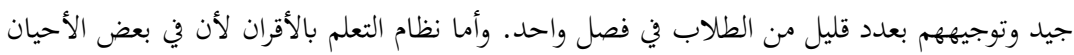
يشعر الطلاب بالتدريس الأفضل بأقرافم من قبل معلمهم.

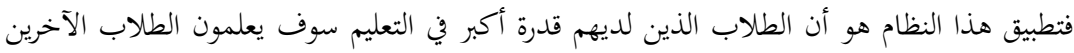

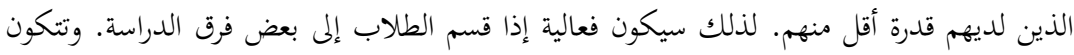

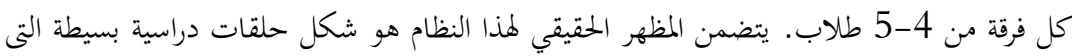

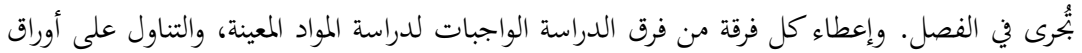

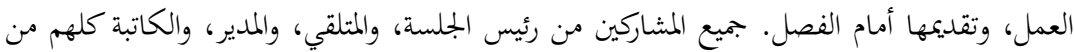


أعضاء الفصل (الطلبة). وأما دور المعلم فيه فقط كمراقب الذي يقوم بالاستناجات والتصحيحات إن وجد الأخطأت. واستنبط من هذين نظامين أن نظام التعليم بها يقال ناجحا. ظهر من أن نظام الفصل الصغير يمكن أن يثير انتباه المعلم لأنماط تعلم الطلاب بشكل فعال وأقصى حد. على سبيل المثال، من خلال القيام على المدخل الفردي حتى يحترم الطلاب كأفراد يختلفون عن بعضهم البعض والطلاب الذين يتمتعون بقدرات أكبر على التعلم سوف يعلمون الطلاب الآخرين الذين لديهم قدرة أقل. د) المواد تعليم اللغة العربية وكان الكتاب المستخدم في تعليم اللغة العربية هو كتاب دروس اللغة العربية بالمدخل العلمي لمنهج 2013، وورقة عملية الطلبة، والكتاب الدعامة "English And Arabic Lessons المخصصة لطلبة فصل تدريب التفوق المؤلف بفريق اللغة عند فصل تدريب التفوق. وإنما استخدام ذالك الكتب ليس لوقت واحد فإنه منفرق بينهما. إن الكتاب اللغة العربية بمنهج 2013 وورقة عملية الطلبة استخدم في الصباح وأما الكتاب English And Arabic Lessons استخدمه في 24. وبنسبة إلى البحث المواد تعليم اللغة العربية باستخدام فصل تدريب التفوق مناسبة بالنظام المرحلة المتوسطة في إندونيسيا واستخدام المواد مناسبة بجزئه. هـ (1) تقييم التعليم إنما التقويمات المستخدمة في فصل تدريب التفوق هما التقييم الشفوي والتقييم التحريري. هناك أربع مهارات اللغوية في تعليم اللغة العربية من الاستماع والكالام والقراءة والكتابة. وفي تطبيقها بالمدرسة

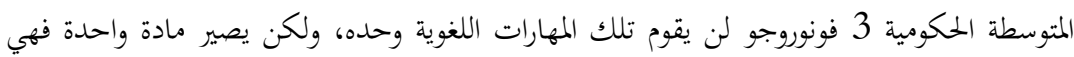
اللغة العربية. إنما إجراء التقييم الشفوي قبل الامتحان التحريري الذي يصبح نقطة تقييم الكلام والمفردات. وفي العادة يقدم المعلم تعليمات للطلاب قبل الامتحان الشفهي ، يقدم المعلم شبكة من الأسئلة أو الأوامر التي سيتم تقديمها أثناء الامتحان الشفهي.

$$
\text { 24 24 خالص، المقابلة 2، المدرسة المتوسطة الإسلامية الحكومية } 3 \text { فونوروغو، } 4 \text { أبريل } 10 \text { ، المدرسة المتوسطة الإسلامية الحكومية } 3 \text { فونوروغو، } 4 \text { أبريل } 2019 .
$$


وأما إجراء التقييم التحريري في هاية مناقشة واحدة أو فصل واحد. وفي العادة يتم إجراء اختبارات كتابية (اختبارات يومية) وأيضًا اختبارات كتابية في فاية الفصل الدراسي (فاية تقييم الفصل الدراسي) أو زيادة في مستوى الدرجات. فإذ وجد الطلاب الذين لم يصلوا إلى حد الأدنى لمعايير الاكتمال، فهناك برنامج علاجي لهم، وإن لم يصلوا حد الأدنى لمعايير الاكتمال فاستعدد على إعطاء الإرشادات الخاصة. بناءً على البحث المذكور، كانت أنشطة التقييم متوافقة بالإجراءات والمراحل التي حددتما الحكومة ومناسبة بالتعليم اللغة العربية لمستوى المدارس المتوسطة. و) البرنامج الداعمة ومن البرنامج الداعمة للتعليم في فصل تدريب التفوق (Kelas Bina Prestasi) في المدرسة المتوسطة الحكومية 3 فونوروجو مما يلى: إصدار الكتاب English And Arabic Lessons، فرقة اللغة العربية، مؤهلات المعلمين والميسرين، اختيار الطلاب، اليوم العربي، المخيم العربي. و. أوجه التشابه والاختلاف بين تعليم اللغة العربية في فصل الثنائية اللغوية في المدرسة المتوسطة الحلكومية 2 فونوروجو وفصل تدريب التفوق في المدرسة المتوسطة الحكومية 3 فونوروجو تؤكد طريقة التعليم المطبقة في هاتين المدرستين (المدرسة المتوسطة الحكومية 2 فونوروجو والمدرسة المتوسطة الحكومية 3 فونوروجو) على أسلوب التعلم النشط الموجه للطلاب. بهذا المدخل، يصير الطالب الممثلين النشطين الذين يبنون المعارف بكل إمكاناهم. وأما دور المعلم العظيم فيه كمسهل وميسر ووسيط. فلم يقم المعلم كموضوع التعليم، ولكن كشريك التعلم عند الطلاب. من خلال النتائج التي حصلت بها الباحثة، هناك أوجه التشابه والاختلاف بين فصل ثنائية اللغة بالمدرسة المتوسطة الحكومية 2 فونوروجو وفصل تدريب التفوق في المدرسة المتوسطة الحكومية 3 فونوروجو، فهو محا يلى: 1. أوجه التشابه في تعليم اللغة العربية في فصل الثنائية اللغوية في المدرسة المتوسطة الحكومية 2 فونوروجو وفي فصل تدريب التفوق في المدرسة المتوسطة الحكومية 3 فونوروجو.

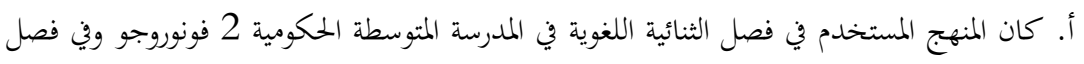
تدريب التفوق في المدرسة المتوسطة الحكومية 3 فونوروجو هو المنهج 2013 بالنهج العلمي. ب. تطبق فصل الثنائية اللغوية في المدرسة المتوسطة الحكومية 2 فونوروجو وفي فصل تدريب التفوق في المدرسة المتوسطة الحكومية 3 فونوروجو نظام مدرسة اليومي الكامل. 
ج. في بجال التعليم، الخاص في تعليم اللغة العربية، استخدمت المواد الدراسي بالكتب المنشر بوزارة

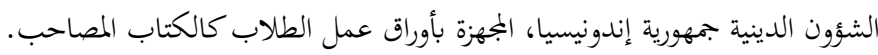

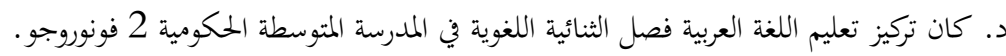

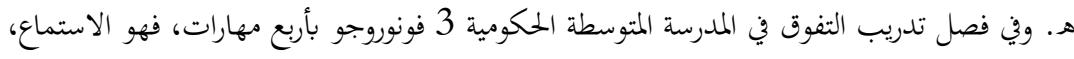

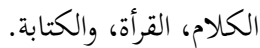
و. من أهداف تعليم اللغة العربية أن يتقن الطلاب المهارت اللغوية الأربعة.

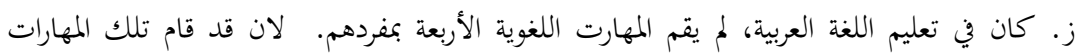

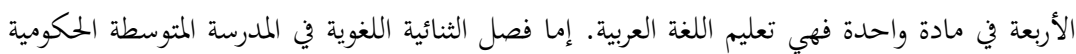
2 فونوروجو أم وفي فصل تدريب التفوق في المدرسة المتوسطة الحكومية 3 فونوروجو. ح. كان طريقة التعليم المطبقة في تعليم اللغة العربية في فصل الثنائية اللغوية في المدرسة المتوسطة

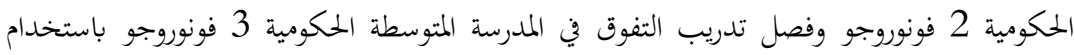

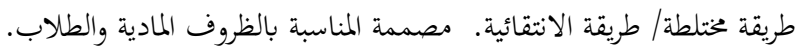

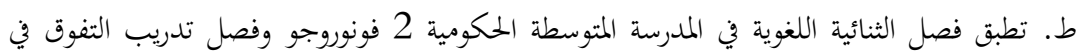

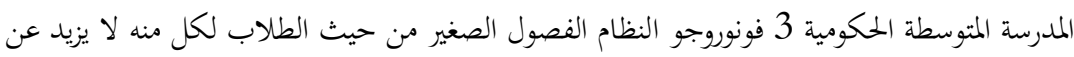
25

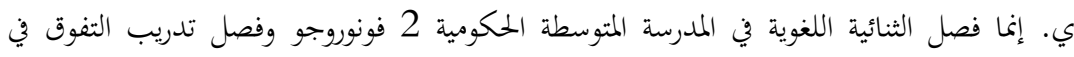
المدرسة المتوسطة الحكومية 3 فونوروجو متكامل بالوسائل الامكانات مثل المكيف الهواء، والشاشاة،

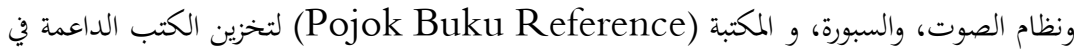

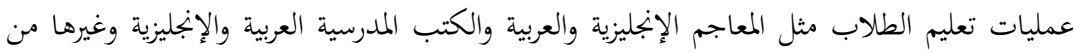

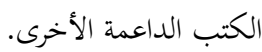

ك. استخدمت أنشطة التقييم في هاتين المدرستين بنفس الطرق، وهي أنشطة التقييم التي قامت بها

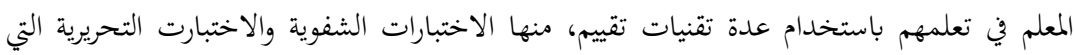
أجريت لتحديد مدى المعرفة يملكها الطلاب. ل. قام فصل الثنائية اللغوية في المدرسة المتوسطة الحكومية 2 فونوروجو وفصل تدريب لمديب التفوق في

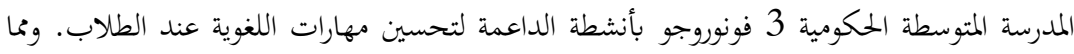
يتعلق بالبرامج الداعمة في هاتين المدرستين، من أن يكون معادلا أم مختلفا، فإنه دليل على أنى أن هاتين 
المدرستين قامت بتنفيذ المنهج المتفوقة لتحسين جودة التعليم حتى يقدر الطلاب على اتقان اللغة العربية

2. الاختلاف في تعليم اللغة العربية في فصل الثنائية اللغوية في المدرسة المتوسطة الحكومية 2 فونوروجو وفصل تدريب التفوق في المدرسة المتوسطة الحكومية 3 فونوروجو. أ. ركز فصل الثنائية اللغوية في المدرسة المتوسطة الحكومية 2 فونوروجو على ترويج اللغة كوسيلة فعالية لإنتاج الأجيال الذين يتقنون اللغات. وكانت اللغة المستخدمة في الفصل هما اللغة الانجليزية واللغة العربية. إنما في تعليم الرياضيات والمواد الدراسية العامة الأخرى يلزم باستخدام اللغة الانجليزية، وأما تعليم العلوم الدينية باستخدام اللغة العربية والاندونيسية وكذلك جميع المواد المستندة إلى تكنولوجيا المعلومات. ويبدأ وقت التعلم من الساعة 6،30 إلى الساعة 13030، مستمر بالتعليم المكثف إلى الساعة 15.30. وقبل الدرس الصباح، بدأ بحفظ المفردات حول 15 دقيقة. ب. استخدم فصل تدريب التفوق في المدرسة المتوسطة الحكومية 3 فونوروجو اللغة الإندونيسية في عملية تعليمه. أما اللغة العربية يُستخدم في عملية تعليم اللغة العربية فحسب، وأحيانا استخدم المعلم والطلاب اللغة الإندونيسية كلغة التواصل في الفصل. قامت عملية تعليم اللغة العربية في فصل تدريب التفوق ثلاث حصص ومزيدة بكصتين في المساء. ج. إن تصميم غرفة فصل الثنائية اللغوية في المدرسة المتوسطة الحكومية 2 فونوروجو معادل بتصميم الفصل العادي.

د. إن تصميم غرفة فصل تدريب التفوق في المدرسة المتوسطة الحكومية 3 فونوروجو بشكل مريح للطلاب، كما إعطاء السجاد فيي غرفة الفصل، وإعدادات المكاتب المتنوعة، مكتب واحد لطالب

$$
\text { ومقعد واحد لطالب واحد. }
$$

هـ ركز فصل الثنائية اللغوية في المدرسة المتوسطة الحكومية 2 فونوروجو بمهارة الكام، إما في الفصل أم البيئة المدرسية، وأما اللغة العربية أم اللغة الانجليزية.

و. كان فصل تدريب التفوق في المدرسة المتوسطة الحكومية 3 فونوروجو يركز بالنظرية الأساسية في عملية تعليم اللغة العربية. ز. إنما فصل الثنائية اللغوية في المدرسة المتوسطة الحكومية 2 فونوروجو ينفذ عملية التعليم المساء بتعليم اللغة العربية لتطوير المهارات اللغوية كما المحادثة والكتابة. 
ح. استخدم فصل تدريب التفوق في المدرسة المتوسطة الحكومية 3 فونوروجو في تعليم اللغة العربية بالكتب المنشر بمعلمي اللغة من فصل تدريب التفوق في المدرسة المتوسطة الحكومية 3 فونوروجو، فهو الكتاب"English and Arabic lesson" الذي يحتوي على المواد المحادثة، والقراءة، والكتابة، والقواعد، والخطابة، والخطط العربية. في هذه الحالة، يسير جهود المدرسة في تحسين أهداف التعليم سيرا حسنا ويحتاج إلى الحفاظ ليقوم بالأهداف الأساسي في عملية التعليم، والخاص في تعليم اللغة العربية. تأمل المدرسة بتوفير الامكانات والتسهيلات المختلفة بالفصول العادية الأخرى لتحسين التعليم الذي يدوم في غرفة الفصل، وكذلك لتحقيق جو موات لتحفيز قدرات الطالاب بأحسن مما يمكن. إنما مادة اللغة العربية مادة الموجه للدعم، والتوجيه، والتطوير، ورعاية المهارات، ولتعزيز الموقف الإيجابي تجاه اللغة العربية من حيث القدرة على فهم خطاب الآخرين وفهم القراءة، والقدرة على استخدام اللغة العربية كوسيلة الاتصال شفهيا وتريريا. وإن المهارات اللغوية العربية وكذلك الموقف الإيجابي باللغة العربية مهمّ جدا للطلاب لمساعدة فهم القرآن الكريم والحديث، وكتب اللغة العربية المتعلقة بالإسلام. من خلال تحليل البيانات العالية يعرف بأن جهود المدرسة والخاصة أولاء المديرين والمعلمين في فصل الثنائية اللغوية في المدرسة المتوسطة الحكومية 2 فونوروجو وفصل تدريب التفوق في المدرسة المتوسطة الحكومية 3 فونوروجو قد دلت على الناحية الإيجابية عند طلبة فصل المتميز الذين قدروا على تظهير الإنجازات المختلفة مثل الفائز الثاني في مسابقة الخطابة العربية في المدرسة العالية الحكومية 2 فونوروجو (طالب من فصل تدريب التفوق في المدرسة المتوسطة الحكومية 3 فونوروجو) العام 2019، والفائز الأول في مسابقة الخطابة من الطالب والطالبة في AKSIOMA على طبقة فرقة عمل المدرسة بالمدرسة المتوسطة الحكومية 2 فونوروجو العام 2019. ز. الحناتمة بالإضافة إلى البيانات من ميدان البحث وقيامت الباحثة بتحليل المشكالات فتصيل الباحثة إلى نتائج البحث كما تلي: تعليم اللغة العربية في فصل الثنائية اللغوية بالمدرسة المتوسطة الحكومية 2 فونوروجو وأما أهداف تعليم اللغة العربية هو تنمية مهارات اللغوية الأربعة وقد صارت مهارة الكلام مراعاة خاصة في هذا الفصل، المناهج تعليم باستخدام المنهج الدّراسي 2013، وإن طريقة تعليم المستخدمة فيه طريقة انتقائية، والمواد تعليم باستخدم هو كتاب اللغة العربية على أساس المنهج الدراسي 2013 من قبل وزارة الشؤون الدينية 
إندونيسيا ، إنما التقويمات المستخدمة التقييم الشفوي والتحريري، وقد صارت مهارة الكلام مراعاة خاصة في هذا الفصل من خلال أنما الانشطة الداعمة فيه كثيرة. تعليم اللغة العربية في فصل تدريب التفوق بالمدرسة المتوسطة الحكومية 3 فونوروجو وأهداف تعليم اللغة العربية لتنمية مهارات الطلبة في اللغة العربية، القراءة وحفظ القرآن الكريم، وتعزيز الطلبة وإعدادهم لممارسة الأولمبياد إما منطقيا أم إقليميا، المناهج تعليم باستخدام المنهج الدّراسي 2013، وإن طريقة تعليم المستخدمة فيه طريقة انتقائية متفق بالمواد وحالات الطلبة، والمواد تعليم باستخدم هو كتاب

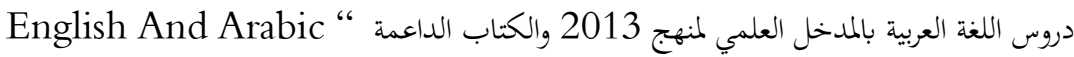

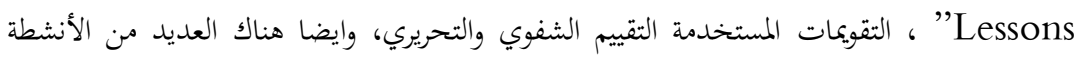
الداعمة لتنمية مهارة اللغة اللعربية في فصل تدريب التفوق. وهناك بعض المعادلات والاختلافات في تعليم اللغة العربية في فصل الثنائية اللغوية وفصل تدريب التفوق. أ) من أوجه التشابه: إنما نظام تعليمه بفصل يوم كامل، وباستخدام منهج 2013، وأهداف تعليمه لدعم الطلبة في اتقان مهارات اللغوية الأربعة إما في فصل الثنائية اللغوية وفصل تدريب التفوق، وإن طريقة تعليم اللغة العربية المستخدمة فيه طريقة انتقائية متفق، وأما التقويم في هاتين المدرستين بالتقويم الشفوي والتقويم التحريري. وكان لمما الانشطة الداعمة الممثلة في ترقية مهارات اللغوية لدي الطلبة. ب) وإنما الاختلافاها: 1) فصل الثنائية اللغوية: بتركيز اللغة كوسيلة فعالة لطباعة الأجيال بمهارة اللغة، وكان اللغة المستخدمة في الفصل هما اللغة الانجليزية والعربية، كان تركيز فصل الدراسي الثنائية اللغوية بمهارة الكلام إما في غرفة الفصل أم في بيئة المدرسة باللغة العربية واللغة الانجليزية، وقام فصل الدراسي الثنائية اللغوية في تعليم اللغة العربية بالمواد كتنمية مهارات اللغوية مثلما المحادثة والكتابة في عملية تعليم المساء. 2) وأما فصل تدريب التفوق: استخدم اللغة الأندونيسية في عملية تعليمه، وكان استخدام اللغة العربية في عملية تعليم اللغة العربية، وفي بعض الأحيان استخدم اللغة الاندونيسية كلغة التواصل في الفصل. تركيزه بالنظرية الأساسية في تعليم اللغة العربية. وقد قام عملية تعليم اللغة العربية في تعليم المساء باستخدام الكتب English and Arabic lesson.

أرشد، أزهر. مدخل إلى طرق تعليم اللغة الأجنبية، أوجونج فاندانج: مطبعة الأحكام، 1998. الحديدي، علي. مشكلة تعليم اللغة العربية لغير العرب، القاهرة: دار الفكر العربي، 2005. 
الحولي، مُحَّه على. الحياة مع لغتين ( الثنائية اللغوية)، مكة :جمع الحكوك تامفوظة للمؤلف، 1988.

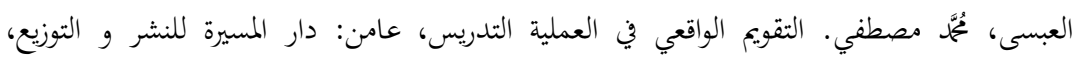
.2010

النعمة، مملوءة. منهج تعليم اللغة العربية في المرحلة المتوسطة، أستاذة في جامعة زين الحسن الإسلامية

$$
\text { غنغوغ فروبولنجوا، 2013. }
$$

الناقة، محمود كامل. تعليم اللغة العربية للناطقين بلغات أخرى، مكة المكرمة: جامعة أم القرى، .1985

بحر الدين، أوريل. تطوير منهج تعليم اللغة العربية و تطبيقه على مهارة الكتابة، مالاغ: مطابع جامعة اسلامية حكومية مالكى الطبعة، 2010.

رسلان، مصطفى. تعليم اللغة العرابية، القاهرة: دار الثقافة للنشر والتوزيع، 2005.

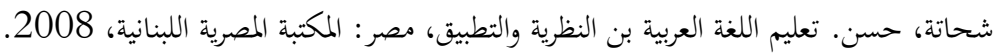

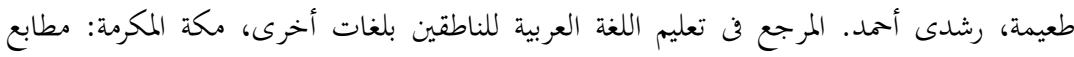
جامعة أم القرى، أمد. 1989.

طعيمة، رشدي أحمد. تعليم العربية لغير الناطقين هما، الرياط: منشورات المنظمة للتربية والعلةم والثقافية

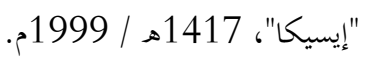

طعيمة، أحمد رشدي ومحمود كامل الناقة. تعليم العربية لغير الناطقين بها، الكتاب الأساس الجزء الأول،

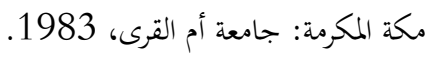

طعيمة، أحمد رشدي. تعليم العربية لغير الناطقين بما مناهجية وأساليبه، مصرى: جامهامعة المنصورة. على الحول، مُمَّمَ. أسالب تدريس اللغة العربية، الرياض :المملكة العربية السعودية، 1982.

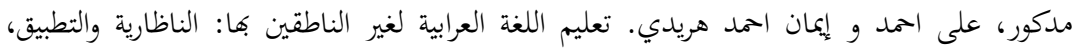
القاهرة :دار الفكر العربي، 2007.

تُحَّمان حفص، منهج تعليم اللغة العربية لطلاب البكالوريوس في قسم تعليم اللغة العربية......، رسالة

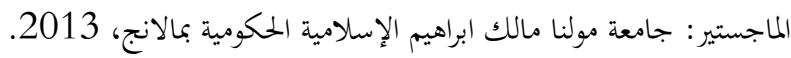
يونس، محمود. التربية والتعليم الجزء الأول "ج"، مطبعة دار السلام، كنتور. Ainin, Muhammad dkk, Evaluasi Pembelajaran Bahasa Arab, (Malang: Misykat, 2006) 
Arikunto, Suharsimi. Prosedur Penelitian Suatu Pendekatan Praktek, Jakarta: PT Rineka Cipta; 2002.

Ayuni, Studi Komparasi Hasil Belajar Antara Siswa Kelas X Program Bina Prestasi Dengan Program Reguler Pada Mata Pelajaran Akidah Akhlak Di MAN 2 Ponorogo tahun ajaran 2012/2013 (Skripsi, STAIN, Ponorogo, 201) 3.

Durus lughoh Arobiyah, buku guru bahasa Arab, pendekatan saintifik kurikulum 2013, Kementerian Agama RI, 2014.

E. Mulyasa, Kurikulum Tingkat Satuan Pendidikan (Bandung: Remaja Rosdakarya, 2006.

Fitri Nursalam, Yufridal. Bahasa Arab (sejarah, Perkembangan, keistimewaan, dan Urgensi Mempelajarinya: Ponorogo ‘ STAIN Ponorogo Press, 2010.

Hadi, Sutrisno. Metodologi Research, Yogyakarta: Penerbit Psikologis Universitas Gajahmada, 1986.

Hermawan, Acep. Metodologi Pembelajaran Bahasa Arab, Bandung: Remaja Rosda Karya, 2012.

Huberman, Myles. Analisis Data Kualitatif, Yogyakarta: Universitas Indonesia, 1992.

Majid, Abdul. Perencanaan Pembelajaran Mengembangkan Standar Kompetensi Guru

Margono. Metodologi Penelitian Pendidikan, Jakarta: Rineka Cipta, 2003.

Moeloeng, Lexy. Metodologi Penelitian Kualitatif, Bandung: PT. Remaja

Rosda Karya, 2000.

Muijs, Daniel, David Reynolds, Effective Teaching Teori dan Aplikasi, ter. Helly prajitno soetjipto dan Sri Mulyantini Soetjipto ( yogyakarta: Pustaka Belajar, 2008.

Munandar, Utami. Pengembangan Kreativitas Anak Berbakat, cet. 3, Jakarta: Departemen Pendidikan Nasional bekerja sama dengan Rineka Cipta, 2009. 
Standar Kompetensi Lulusan (SKL), Standar Kompetensi (SK) dan Kompetensi Dasar (KD) serta Model Pengembangan Silabus Madrasah Tsanawiyah (Mata Pelajaran Bahasa Arab), Direktorat Pendidikan Madrasah, Direktorat Jenderal Pendidikan Islam Depertemen Agama RI: 7111

Tim Penyusun Kamus Pusat Bahasa, Kamus Besar Bahasa Indonesia, 1995

http://afrizaldaonk.blogspot.com/2011/01/blog-post_3796.html http://sastra.um.ac.id/wp-content/uploads/2009/10/

Penyelarasan-Materi-dan-Model-RPP-Bahasa-

Arabuntuk-Pendidikan-Dasar-dan-Menengah-Nurul-

Murtadho.pdf, diakses 20-15-2019.

http://www.alriyadh.com/2011/02/01/article600258.html http://www.kau.edu.sa/content.aspx?Site_ID $=\& \operatorname{lng}=$ AR\&cid $=2$ 7403. 